G. Frerichs: Salpetersüurebestimmung im Wasser.

\begin{tabular}{lrrrcc} 
& \multicolumn{2}{c}{ Gefunden: } & \multicolumn{3}{c}{ Berechnet für } \\
& 1. & 2. & $\mathrm{C}_{14} \mathrm{H}_{12} \mathrm{O}:$ & $\mathrm{C}_{\mathbf{1 8}} \mathrm{H}_{10} \mathrm{O}:$ & $\mathrm{C}_{18} \mathrm{H}_{18} \mathrm{O}:$ \\
$\mathrm{H}$ & 6,1 & 6,0 & 6,12 & 5,53 & 6,57 \\
$\mathrm{C}$ & 85,2 & 85,3 & 85,71 & 85,67 & 84,74 \\
$\mathrm{O}$ & 8,7 & 8,7 & 8,16 & 8,79 & 8,69 \\
M.-G. 188,4 & & 196 & 182 & 184.
\end{tabular}

Wir haben dann noch versucht die Natur des Sauerstoffes zu ergründen, indem wir auf Fraktion II Phenylisocyanat, Natriumbisulfit, Hydroxylamin, Semikarbazon, Amidoguanidin etc. einwirken ließen. Alle Versuche waren ohne Erfolg, so daß wir nicht in der Lage sind, nähere Angaben über die Bindungsweise des Sanerstoffes zu machen. Ebensowenig vermögen wir etwas über die Natur des Kohlenstoffkernes anszusagen; das hohe spezifische Gewicht und der niedrige Wasserstoffgehalt deuten allerdings auf einen aromatischen Komplex, doch mu@ es späteren Untersuchungen überlassen bleiben, die Natur des interessanten Körpers aufzuklären. Wir gedenken zunächst die von der Firma Heinrich Haensel in Aussicht gestellten weiteren Mitteilungen abzuwarten, bevor wir Anstalten zur Fortführung unserer Untersuchungen treffen werden.

Dezember 1902.

Pharm.-chem. Institut Marburg und Breslau.

Mitteilung sus dem pharm.-chemischen Laboratorium der technischen Hochschule in Braunschweig. Von H. Beckurts.

\title{
Ein einfaches Verfahren zur quantitativen Bestimmung der Salpetersäure im Wasser.
}

Von G. Frerichs.

Die zur Bestimmung der Salpetersäure im Wasser bisher angewandten Methoden sind entweder nicht sebr genau, oder ibre Ausführung ist zu umständlich. Letzteres gilt namentlich für die gasometrische Methode nach Schulze-Tiemann. Die kolorimetrischen 
Bestimmmungen lassen zwar an Einfachheit nichts za wünschen übrig, die Genauigkeit ist aber keine besonders hervorragende. Dasselbe trifft auch für die Indigomethode zu.

Im nachstehenden soll nun eine Methode beschrieben werden, welche es ermöglicht, die Salpetersäure im Wasser auf mühelose Weise mit derselben Genauigkeit zu bestimmen, mit welcher die Chloride durch Titration mit Silbernitratlösung bestimmt werden.

Das Verfahren beruht einfach darauf, daß sich die im Wasser vorkommenden Nitrate durch Salzsäure sehr leicht in Chloride verwandeln lassen und $d a \beta$ ein Ueberschu $B$ an Salzäure schon beim Abdampfen anf dem Wasserbade entfernt wird.

Man hat also weiter nichts nötig, als den ursprünglichen Gehalt an Chloriden zu titrieren, mit Salzsäure zur Trockne einzudampfen und wieder mit Silbernitrat zu titrieren. Die nach Abzug der ursprünglichen Menge verbleibende Menge des Chlors entspricht dann dem vorhanden gewesenen Gehalt an Salpetersäure. Vorausgesetzt ist hierbei natïrlich die Entfernung der Karbonate, da die Kohlensäure ebenfalls durch Chlor ersetzt wird und als Salpetersäure mit bestimmt werden würde.

Um die Karbonate des Calciums und Magnesiums, sowie auch Eisen und Aluminiumverbindungen und die geringen Mengen von Silikaten zu entfernen, hat man nur nötig das Wasser zur Trockne zu verdampten und den Abdampfrückstand, dessen Menge man in der Regel ja anch bestimmt, mit Wasser zu behandeln. Man kann dann das Filtrat, welches die Gesamtmenge der Chloride und Nitrate und einen Teil der Sulfate enthält, zur Bestimmung der Salpetersäure ohne weiteres benutzen, indem man dasselbe mit Salzsäure eindampft und den Rückstand mit Silbernitrat titriert.

Zur Entfernung von Alkalikarbonaten, welche aber nur höchst selten und dann nur in geringer Menge vorkommen, fügt man dem Wasser eine kleine Menge Chlorcalcium oder Chlorbaryum hinzu, bestimmt die nun vorhandene Menge Chlor, dampft zur Trockne ein und verwendet den kalten Auszug des Abdampfrückstandes zur Bestimmung der Salpetersäure.

Ist die ursprünglich vorhandene Menge an Chlor sehr groß, etwa mehr als $30 \mathrm{mg}$ in $100 \mathrm{ccm}$ Wasser - ein Fall der selten vorkommen dürfte - so ist es zweckmäßig, den größten Teil der Chloride in Sulfate zu verwandeln, indem man das Wasser mit Silbersulfat behandelt. Um $10 \mathrm{mg}$ Chlor zn entfernen, sind theoretisch $43,9 \mathrm{mg}$ Silbersulfat erforderlich. Da es nun von Nachteil ist, wenn das Wasser Silber im Ueberschuß enthält, so darf man nicht die ganze erforderliche Menge 
an Silbersulfat anwenden, sondern es empfiehlt sich, auf je $1 \mathrm{mg}$ Chlor $4 \mathrm{mg}$ Silbersulfat dem Wasser zuzusetzen, es bleibt dann etwa der 10. Teil des Chlors im Wasser zurück. Man fübrt die Behandlung eines Wassers mit Silbersulfat einfach in der Weise aus, daß man zunächst die Menge des Chlors bestimmt, dann $300 \mathrm{ccm}$ des Wassers mit dem vierfachen des in dieser Menge enthaltenen Chlors an trockenem zerriebenen Silbersulfat versetzt und unter öfterem Umschütteln einige Zeit an einem warmen Ort stellt. Nach dem Abfiltrieren des Chlorsilbers bestimmt man in einem Teil des Filtrates die Menge des noch vorhandenen Chlors und in einem anderen Teil die Salpetersäure.

Die Umwandlung der Chloride in Sulfate ist nicht unbedingt notwendig, sie ist aber bei Gegenwart nur kleiner Mengen von Salpetersäure deswegen vorteilhaft, weil die zur Erzielung genauer Resultate nötige Menge Wasser unverhältnismäßig viel Silberlösung zur Bindung des ursprünglichen Chlors erfordern würde. Sehr zu empfehlen ist die Entfernung der größten Menge des Chlors dann, wenn dasselbe an Magnesium gebunden ist, weil größere Mengen Chlormagnesium eine vorsichtigere Ausführung der Bestimmung bedingen.

Bei der Bestimmung der Salpetersänre nach dem oben erläuterten Prinzip ist die Beschaffenheit der zur Verwendung gelangenden Salzsäure zu berücksichtigen, denn selbst die reinste Säure hinterläßt beim Abdampfen größerer Mengen einen Rückstand, welcher bestimmbare Mengen von Chloriden enthält. Um die dadurch bedingten Fehler auszuscheiden, ist es nicht etwa nötig, jedesmal eine frisch destillierte Salzsäure zu verwenden, sondern man braucht nur einen blinden Versuch auszuführen, indem man die gleiche Menge an Salzsäure, welche man bei der Bestimmung der Salpetersäure verwendet, in einer Porzellanschale verdampft und das Chlor in dem Rückstande bestimmt.

Da die Umsetzung zwischen Nitraten und Chlorwasserstoff ein Prozeß ist, welcher nicht im einfachen stöchiometrischen Verhältnisse vor sich geht, sondern nur dann im gewünschten Sinne verläuft, wenn die Menge des Chlorwasserstoffes diejenige der Nitrate um ein Vielfaches übertrifft, so ist zur vollständigen Zersetzung der Nitrate ein erheblicher Ueberschuß an Salzsäure erforderlich '). So genügte einmaliges Abdampfen von $0,1 \mathrm{~g}$ Kalisalpeter mit $20 \mathrm{ccm}$ Salzsäure nicht zur vollständigen Entfernung der Salpetersäure; nach nochmaligem Eindampfen mit $20 \mathrm{ccm}$ Salzsäure war jedoch keine Salpetersäure mehr nachzuweisen, $50 \mathrm{ccm} 25 \%$ iger Salzsäure werden also auf jeden Fall zur Zersetzung von 0,1 $\mathrm{g}$ Salpeter ausreichen. In $50 \mathrm{ccm}$ verschiedener

1) Umgekehrt kann man durch Salpetersäure Chloride wieder in Nitrate verwandeln.

Arch. d. Pharm. CCXXXXI. Bds. 1. Heft. 
Salzsäuren wurden nun $0,5-2,3 \mathrm{mg}$ Chlor im Rückstande gefunden, eine Menge, welche bei einer Salpetersäurebestimmung erhebliche Fehler bedingen würde und deshalb auf jeden Fall in Rechnung zu setzen ist.

Um nun eine Bestimmung der Salpetersäure im Wasser auszuführen, orientiert man sich zunächst über die Menge der vorhandenen Salpetersäure, wozu die Diphenylaminreaktion genügt. Erhält man eine starke Blaufärbung, so genügen zur Bestimmung $100 \mathrm{ccm}$ des Wassers, ist die Reaktion nur schwach, so verwendet man eine entsprechend größere Menge. Das Wasser wird zur Trockne verdampft, der Rückstand mit Wasser behandelt und das Unlösliche abfiltriert. Das Filtrat wird dann mit $50 \mathrm{ccm}$ Salzsäure in einer Porzellanschale auf dem Wasserbade zur Trockne verdampft, bis kein stechender Geruch mehr bemerkbar ist. Hierbei hat man auch darauf zu achten, dal am inneren Rande der Schale kein feuchter Beschlag sich befindet, weil ein solcher noch Salzsäure enthalten würde. Zur Sicherheit kann man auch die Schale noch einige Zeit in einem Trockenschrank bis $100^{\circ}$ erhitzen. Darauf löst man den Abdampfrückstand in etwa $30-50 \mathrm{ccm}$ Wasser und überzeugt sich nun zunächst davon, daß alle Salpetersäure entfernt ist, indem man einen Tropfen der Lösung mit Diphenylaminschwefelsäure zusammenbringt. In den seltensten Fällen wird ein nochmaliges Eindampfen mit Salzsäure erforderlich sein.

Ist keine Salpetersäure mehr nachweisbar, so titriert man direkt in der Porzellanschale mit Silbernitrat unter Anwendung von Kaliumchromat als Indikator. Gleichzeitig verdampft man $50 \mathrm{ccm}$ derselben Salzsäure zur Trockne and bestimmt im Rückstande das Chlor. Zur Titration verwendet man zweckmäßig eine Silberlösung, welche 4,8 g Silbernitrat im Liter enthält, und von welcher jedes Kubikzentimeter $1 \mathrm{mg}$ Chlor entspricht. Von der im Rückstande gefundenen Menge Chlor subtrahiert man nun diejenige Menge, welche ursprünglich im Wasser enthalten war und die für den Rückstand der Salzsäure gefundene. Der Rest wird dann zur Ermittelung der vorhanden gewesenen Salpetersäure $\left(\mathrm{N}_{2} \mathrm{O}_{5}\right)$ mit 1,525 multipliziert, da $1 \mathrm{mg}$ Chlor $1,525 \mathrm{mg} \mathrm{N} \mathrm{N}_{2} \mathrm{O}_{3}$ entspricht. Bei der Verwendung von $1 / 100 \mathrm{~N}$.-Silbernitratlösung berechnet man ebenfalls zunächst die Menge des für die Salpetersäure eingetretenen Chlors und multipliziert diese mit 1,525.

Zum Beweise der Zuverlässigkeit der oben beschriebenen Methode wurden folgende Versuche ausgeführt:

1. $50 \mathrm{ccm}$ einer Lösung von $1 \mathrm{~g} \mathrm{KNO}_{3}$ in 1 Liter wurden mit $2 \times 20 \mathrm{ccm} 25 \%$ iger Salzsäure eingedampft und das Chlor im Rückstand titriert. (Nach einmaligem Eindampfen mit $20 \mathrm{ccm}$ Salzsăure war noch eine geringe Spur Salpetersăure nachzuweisen.) 
Es wurden im Rückstande gefunden $19,0 \mathrm{mg} \mathrm{Cl}$

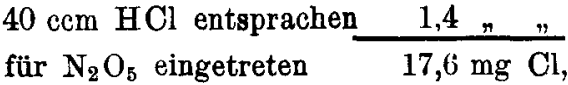

$17,6 \mathrm{mg} \mathrm{Cl}=26,84 \mathrm{mg} \mathrm{N} \mathrm{N}_{2} \mathrm{O}_{5}, 50 \mathrm{ccm}$ ¿der Lösung $=0,05 \mathrm{~g} \mathrm{KNO}_{8}$ entsprechen $26,73 \mathrm{mg} \mathrm{N}_{3} \mathrm{O}_{5}-$, die gefundene Menge stimmte also mit der angewandten sehr gut überein. Zwei gleiche Versuche lieferten genau dasselbe Resultat.

2. $100 \mathrm{ccm}$ der Salpeterlösung wurden mit $70 \mathrm{ccm}$ Salzsäure eingedampft:

\begin{tabular}{lc} 
Chlor im Rückstand & $37,4 \mathrm{mg}$ \\
für $70 \mathrm{ccm} \mathrm{HCl}$ & $2,45 \%$ \\
\cline { 2 - 2 } & $\begin{array}{c}34,95 \mathrm{mg} \\
\text { für } \mathrm{N}_{2} \mathrm{O}_{8}\end{array}$
\end{tabular}

$34,95 \mathrm{mg} \mathrm{Cl}=53,30 \mathrm{mg} \mathrm{N} \mathrm{N}_{5}$, angewandt $53,46 \mathrm{mg} \mathrm{N} \mathrm{N}_{2} \mathrm{O}_{5}$.

3. $25 \mathrm{ccm}$ der Salpeterlösung wurden mit $20 \mathrm{ccm}$ Salzsăure eingedampft:

$\begin{array}{ll}\text { Chlor im Rückstand } & 9,45 \mathrm{mg} \\ \text { für } 20 \mathrm{ccm} \mathrm{HCl} & 0,7 \mathrm{~m} \\ \text { für } \mathrm{N}_{2} \mathrm{O}_{5} & 8,75 \mathrm{mg},\end{array}$

$8,75 \mathrm{mg} \mathrm{Cl}=13,34 \mathrm{mg} \mathrm{N}_{2} \mathrm{O}_{5}$, angewandt $13,36 \mathrm{mg} \mathrm{N}_{2} \mathrm{O}_{5}$.

4. $20 \mathrm{ccm}$ einer Chlormagnesiumlösung, welche in $100 \mathrm{ccm} 69 \mathrm{mg}$ Chlor enthielt, wurden auf dem Wasserbade zur Trockne verdampft; der Rückstand enthielt $13,8 \mathrm{mg}$ Chlor, es war also kein Verlust an Chlor eingetreten.

Einige weitere in derselben Weise ausgeführte Versuche lieferten die gleichen Resultate, sodaß also beim Eindampfen von chlormagnesiumhaltiger Lösung auf dem Wasserbade ein Verlust an Chlor nicht zu befürchten ist.

5. $50 \mathrm{ccm}$ der Salpeterlösung (1:1000) wurden mit $25 \mathrm{ccm}$ der Chlormagnesiumlösung $(69 \mathrm{mg} \mathrm{Cl}$ in $100 \mathrm{ccm})$ und $50 \mathrm{ccm}$ Salzsäure (2,0 mg Cl im Rückstand) zur Trockne verdampft:

\begin{tabular}{|c|c|}
\hline $\begin{array}{l}\text { Chlor im Rückstand } \\
\text { für } 50 \mathrm{ccm} \mathrm{HCl}\end{array}$ & $\begin{array}{c}36,7 \mathrm{mg} \\
2,0\end{array}$ \\
\hline $25 \mathrm{ccm} \mathrm{MgCl}_{2}$ & $\begin{array}{l}34,7 \mathrm{mg} \\
17,25\end{array}$ \\
\hline $\mathrm{N}_{2} \mathrm{O}$ & $17,45 \mathrm{mg}$ \\
\hline
\end{tabular}

$17,45 \mathrm{mg} \mathrm{Cl}=26,61 \mathrm{mg} \mathrm{N}_{2} \mathrm{O}_{5}$, angewandt $26,73 \mathrm{mg} \mathrm{N}_{2} \mathrm{O}_{5}$.

6. $25 \mathrm{ccm}$ Salpeterlősung wurden mit $50 \mathrm{ccm}$ Chlormagnesium. lösung und $50 \mathrm{ccm}$ Salzsäure eingedampft: 


\begin{tabular}{lc} 
Chlor im Rückstand & $41,4 \mathrm{mg}$ \\
für $50 \mathrm{ccm} \mathrm{HCl}$ (neue Săure) & $1,6 \quad$. \\
\cline { 2 - 2 } & $39,8 \mathrm{mg}$ \\
für $50 \mathrm{ccm} \mathrm{Mg} \mathrm{Cl}_{2}$ & $34,5 \mathrm{~N}$ \\
für $\mathrm{N}_{2} \mathrm{O}_{5}$ & $5,3 \mathrm{mg}$,
\end{tabular}

$5,3 \mathrm{mg} \mathrm{Cl}=8,18 \mathrm{mg} \mathrm{N} \mathrm{N}_{2} \mathrm{O}_{5}$, angewandt $13,36 \mathrm{mg} \mathrm{N} \mathrm{N}_{2} \mathrm{O}_{5}$.

Die gefundene, ganz erhebliche Differenz erklärt sich dadurch, daß beim Eindampfen der Chlormagnesium enthaltenden Flüssigkeit das Wasserbad auggekocht war, und durch die dadurch bedingte höhere Temperatur eine Zersetzung des wasserhaltigen Chlormagnesiums in Magnesiumoxychlorid und Salzsäure eingetreten war. Der Versuch wurde nur deswegen zu Ende geführt, um den Einfluß größerer Menge von Chlormagnesium bei höherer Temperatur kennen zu lernen. Eine Wiederherstellung der normalen Bedingung dieses Versuches wäre sehr einfach durch nochmaliges Eindampfen mit Salzsäure bei Wasserbadtemperatur zu erzielen gewesen; der Versuch lehrt aber, daß ein großer Gehalt an Chlormagnesium unter Umständen störend wirken kann, und daß deshalb eine Entfernung des Chlors bei Gegenwart größerer Menge von Chloriden durch Silbersulfat vorteilhaft ist.

7. $100 \mathrm{ccm}$ Salpeterlösung und $200 \mathrm{ccm}$ Chlormagnesiumlösung $(=138 \mathrm{mg} \mathrm{Cl})$ wurden mit $0,55 \mathrm{~g}$ Silbersulfat in der Wärme geschüttelt und abfiltriert, bevor alles Silbersulfat in Lösung gegangen war. $100 \mathrm{ccm}$ des Filtrates enthielten $26,8 \mathrm{mg}$ Chlor.

$100 \mathrm{~cm}$ des Filtrates wurden mit $50 \mathrm{ccm}$ Salzsäure eingedampft:

\begin{tabular}{|c|c|}
\hline $\begin{array}{l}\text { Chlor im Rückstand } \\
\text { für } 50 \mathrm{ccm} \text { Salzsäure (neue }\end{array}$ & $\begin{array}{c}38,8 \mathrm{mg} \\
0,5 \quad\end{array}$ \\
\hline agliches Chlor & $\begin{array}{l}38,3 \mathrm{mg} \\
26,8 \mathrm{~m}\end{array}$ \\
\hline $\mathrm{N}_{2} \mathrm{O}_{5}$ & $11,5 \mathrm{mg}$ \\
\hline
\end{tabular}

$11,5 \mathrm{mg} \mathrm{Cl}=17,54 \mathrm{mg} \quad \mathrm{N}_{2} \mathrm{O}_{5}$, angewandt $17,82 \mathrm{mg} \mathrm{N}_{2} \mathrm{O}_{5}$ (1/3 von $100 \mathrm{ccm}$ Lösung).

Andere Chloride, wie Chlornatrium, Chlorkalium und auch Chlorcalcium üben auf die Bestimmung keinen Einfluß aus, größere Mengen derselben lassen sich durch Silbersulfat gegebenenfalls leicht, beseitigen.

8. $100 \mathrm{ccm}$ eines salpetersäurefreien Leitungswassers, welche $3,5 \mathrm{mg}$ Chlor enthielten, wurden mit $25 \mathrm{~cm}$ der Salpeterlösung versetzt, zur Trockne verdampft und der wässerige Auszug des Rückstandes mit $50 \mathrm{ccm}$ Salzsäure eingedampft: 
G. Frerichs: Salpetersäurebestimmung im Wasser.

\begin{tabular}{|c|c|c|}
\hline $\begin{array}{l}\text { Chlor im Rückstand } \\
\text { für } 50 \mathrm{ccm} \mathrm{HCl}\end{array}$ & & $\begin{array}{c}13,9 \mathrm{mg} \\
1,6 \quad n\end{array}$ \\
\hline gliches $C$ & Chlor & $\begin{array}{c}12,3 \mathrm{mg} \\
3,5,\end{array}$ \\
\hline${ }_{2} \mathrm{O}$ & & \\
\hline
\end{tabular}

$8,8 \mathrm{mg} \mathrm{Cl}=13,42 \mathrm{mg} \mathrm{N} \mathrm{N}_{2} \mathrm{O}_{5}$, angewandt $13,36 \mathrm{mg} \mathrm{N}_{2} \mathrm{O}_{5}$.

9. $100 \mathrm{ccm}$ salpetersäurefreies Flußwasser mit $10,5 \mathrm{mg}$ Chlor wurden mit $50 \mathrm{cem}$ Salpeterlösung versetzt und in der unter 8 beschriebenen Weise weiter behandelt:

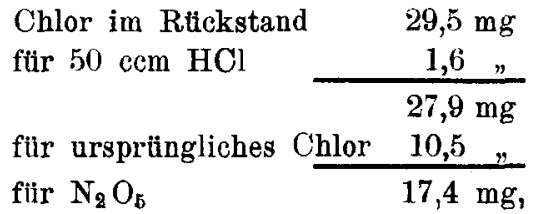

$17,4 \mathrm{mg}$ Chlor $=26,53 \mathrm{mg} \mathrm{N} \mathrm{N}_{2} \mathrm{O}_{5}$, angewandt $26,73 \mathrm{mg} \mathrm{N} \mathrm{N}_{2} \mathrm{O}_{5}$.

Aus den beschriebenen Versuchen geht hervor, daß das Verfahren Resultate liefert, deren Genauigkeit von keiner anderen Methode utbertroffen werden dürfte. An Einfachheit läßt das Verfahren ebenfalis nichts zu wünschen übrig, da das Eindampfen des Wassers, das Ausziehen des Rückstandes mit Wasser und das Wiedereindampfen mit Salzsäure nur wenig Mühe verursacht.

$\mathrm{Daß}$ nach dieser Methode die salpetrige Säure als Salpetersäure mit bestimmt wird, ist ein nur kleiner Fehler, welcher auch fast allen anderen Methoden anhaftet. Die Menge der im Wasser zuweilen vorkommenden salpetrigen Säure ist aulerdem meistens so gering, daß die Bestimmung der Salpetersäure dadurch kaum beeinflußt wird.

Bei Ausflihrung der Versuche bin ich in dankenswerter Weise durch Herrn Apotheker H. Hollandt unterstätzt worden. 